\title{
3 Research Square \\ Small Bowel Radiographic Imaging is Not Useful for Isolated Iron-Deficiency Anemia
}

Richard Hae

University of Ottawa https://orcid.org/0000-0001-6630-3168

Sanjay Murthy ( $\nabla$ smurthy@toh.ca )

https://orcid.org/0000-0002-0128-7897

Research article

Keywords: Small bowel imaging; Iron deficiency anemia; Computed tomography enterography; Esophagogastroduodenoscopy; Colonoscopy; Occult gastrointestinal bleeding

Posted Date: September 5th, 2019

DOI: https://doi.org/10.21203/rs.2.13916/v1

License: (c) (1) This work is licensed under a Creative Commons Attribution 4.0 International License. Read Full License 


\section{Abstract}

Background Iron deficiency anemia in the absence of overt bleeding can result from chronic occult blood loss from the gastrointestinal tract. When esophagogastroduodenoscopy (EGD) and colonoscopy fail to demonstrate clinically significant lesions (CSLs), practitioners often consider small bowel investigations, such as CT enterography and video capsule endoscopy. Although most society guidelines recommend further investigations to evaluate the small bowel, few studies have evaluated the utility of small bowel radiographic imaging in this setting.Methods This is a retrospective cross-sectional study investigating the rates and types of CSLs identified during EGD, colonoscopy and small bowel imaging among individuals over age 50 who were referred for new-onset iron deficiency anemia without overt bleeding. Data was collected on 334 consecutive patients who underwent colonoscopy with or without EGD to evaluate new-onset iron deficiency anemia at The Ottawa Hospital from January 1, 2010 to March 30, 2012. We evaluated the frequency of CSLs among all patients and those who further underwent small bowel radiographic imaging. Results Of the 332 individuals who underwent colonoscopy, 42 (12.7\%) were found to have a CSL, with 37 of these being malignancy or high-grade adenomas. Of the 258 individuals who also underwent EGD, 49 (19.0\%) had CSLs, including 11 vascular ectasias, 15 peptic ulcer disease, 8 celiac disease, 8 severe erosive esophagitis, and 9 esophageal varices. Of 243 patients who did not have any CSLs identified on colonoscopy +/- EGD, 75 underwent subsequent small bowel imaging, and 9 patients underwent small bowel imaging despite having a CSL found on endoscopy. A total of 84 patients underwent subsequent small bowel imaging (68 CT enterography, 13 small bowel followthrough, one MR enterography, one CT enteroclysis, and one push enteroscopy). In these 84 patients, only one relevant CSL was found (inflammatory bowel disease).Conclusions Upper and lower endoscopy are valuable tools for diagnosing gastrointestinal tract lesions in individuals over the age of 50 with newly diagnosed iron deficiency anemia in the absence of overt bleeding. CT enterography and small bowel follow-through have limited utility in this setting.

\section{Introduction}

Iron-deficiency anemia (IDA) in the absence of overt rectal bleeding often results from chronic occult blood loss or impaired iron absorption from the gastrointestinal tract (GIT). Many gastrointestinal conditions may predispose to IDA, including GIT cancers (most commonly colorectal cancer and gastric cancer), inflammatory bowel diseases (IBD), celiac disease, peptic ulcer disease (PUD), erosive esophagitis or gastritis, chronic atrophic gastritis and vascular ectasias, most of which carry long-term health risks in the absence of definitive treatment $[1,2,3]$. The vast majority of these disorders occur primarily in either the proximal (proximal to the ligament of Treitz) or the distal GIT (terminal ileum and/or colon) [1]. Therefore, in the absence of another apparent cause for IDA, it is standard to perform structural investigations of these areas, including colonoscopy (with intubation of the terminal ileum) and esophagogastroduodenoscopy (EGD), with the choice of initial investigation guided by the clinical history [4]. Historical studies have reported rates of clinically significant lesions (CSLs) found on EGD and colonoscopy of $40-70 \%$ during investigation of IDA $[5,6]$. 
When EGD and colonoscopy fail to demonstrate CSLs that account for asymptomatic IDA, practitioners must consider whether to further investigate the deeper small bowel. This often requires tests that are not routinely accessible, invasive, resource intensive and/or expose patients to ionizing radiation, including deep balloon enteroscopy (DBE), video capsule endoscopy (VCE), computed tomographic enterography (CTE) and magnetic resonance enterography (MRE) $[7,8]$. While historical studies have reported rates of CSLs in the small bowel in the context of IDA ranging from $32 \%$ to $78 \%$ [9] following negative EGD and colonoscopy, most of these studies included patients with overt and occult bleeding. The few studies that have specifically evaluated obscure occult IDA cite much lower rates of positive findings, with MR enterography being normal in $92.5 \%$ of subjects in one study [10]. Nevertheless, many practitioners may feel compelled to proceed with small bowel investigations, particularly in elderly individuals, for fear of missing small bowel cancers, Crohn's disease or vascular ectasias that are at risk of re-bleeding. The reported prevalence of small bowel cancers in the general population, including adenocarcinoma, lymphoma, stromal tumours and others, is 1 to 1.5 per 100,000 persons $[11,12]$.

Societal guidelines provide variable recommendations regarding investigation of the small bowel in individuals with asymptomatic IDA. Most recommendations group these individuals together with those who have overt GIT bleeding. The American College of Gastroenterology (ACG) and European Society of Gastrointestinal Endoscopy (ESGE) recommend VCE in all patients with IDA following negative upper and lower endoscopy, with or without overt bleeding, whereas the Canadian Association of Gastroenterology (CAG) recommends small bowel imaging in select individuals $[13,14,15]$. VCE is universally recommended as the first-line test in this setting $[13,14,15]$. However, VCE is not readily available in many institutions and the cost is not always re-imbursed by third-party payers. Therefore, radiographic tests, such as CTE and MRE, are frequently substituted as first-line tests to evaluate the small bowel in this context. Notably, the yield of CTE and MRE are reported to be considerably inferior to VCE for diagnosing small bowel lesions, albeit not all lesions identified on VCE might be considered clinically significant in these studies $[16,17,18]$.

Overall, there remains considerable uncertainty regarding the appropriate investigation of asymptomatic IDA, and particularly the value of small bowel imaging, in persons with who have a negative EGD and colonoscopy. As IDA occurs in up to $12 \%$ of people in industrialized countries and $45 \%$ in nonindustrialized countries, gastrointestinal investigations in these patients potentially represent a significant source of health care resource utilization [19]. Therefore, we assessed the frequency and nature of CSLs (i.e. those potentially requiring therapy) observed during EGD, colonoscopy and small bowel imaging studies in the investigation of persons aged 50 years or older with IDA in the absence of overt bleeding at our referral center.

\section{DATA SOURCES}

We identified persons aged 50 years or older who underwent colonoscopy at The Ottawa Hospital using the Ottawa Hospital Data Warehouse, a repository of clinical, laboratory, imaging and pathological information for all patient encounters at The Ottawa Hospital. We conducted a detailed chart review in all 
patients to identify those who had received a complete colonoscopy (i.e. intubation of cecum or terminal ileum) with a reported adequate bowel preparation, as well as to identify procedural indications and CSLs on endoscopy, based on endoscopy and pathology reports.

\section{Materials And Methods}

We conducted a retrospective cross-sectional study in 334 consecutive patients aged 50 years or older who underwent colonoscopy for evaluation of physician-reported new-onset or undiagnosed IDA at The Ottawa Hospital between January 1, 2010 and March 30, 2012. To fully capture patients encompassing the spectrum of IDA, we also included persons with iron deficiency in the absence of anemia or a measured hemoglobin level at the time of evaluation, as well as those with microcytic anemia that was suspected to be related to iron deficiency by the treating physician. We excluded patients who had overt GIT bleeding, those who had previously undergone GIT investigations for IDA and those who already had an established cause for IDA.

In this cohort, we evaluated the frequency of performance of EGD and small bowel imaging studies, as well as the frequency and nature of CSLs that would indicate a need for treatment. Small bowel investigations included CT enterography, MR enterography, capsule endoscopy, CT enteroclysis, push enteroscopy, and small bowel follow-through. We defined CSLs in colonoscopy as any of malignancy, high grade adenomas (defined as $\geq 3$ adenomas, adenoma $>1 \mathrm{~cm}$, or adenomas with villous, serrated or high-grade dysplastic features), IBD, moderate-severe vascular ectasias and large rectal varices. We defined CSLs in EGD as any of malignancy, bleeding polyps > $1 \mathrm{~cm}$, IBD, moderate-severe vascular ectasias, PUD, severe erosive esophagitis, esophago-gastric varices, severe portal gastropathy, severe atrophic gastritis, and celiac disease. We defined CSLs in small bowel imaging studies as any of neoplasms, IBD and large vascular ectasias. We also collected information on patient co-morbidities (using the Charlson index) [20], concurrent symptoms at presentation (weight loss, altered bowel habits, abdominal pain, dyspepsia), and proton pump inhibitor use at the time of endoscopy.

All data are reported descriptively. The primary outcome was the proportion of patients who had CSLs on small bowel imaging following a negative EGD and colonoscopy. Secondary outcomes were the rates of CSLs on EGD and colonoscopy. We also evaluated practice patterns at our center, including the proportion of patients who underwent planned colonoscopy and EGD on the same day, as well as the proportion of persons who underwent small bowel imaging following negative or positive endoscopy.

\section{Results}

Baseline characteristics of study patients are summarized in Table 1. Of 334 consecutive patients who underwent colonoscopy +/- other investigations for a primary indication of IDA, 101 had at least one other presenting complaint at the time of referral. 258 patients underwent EGD (77.2\%), while 198 (59.3\%) underwent both EGD and colonoscopy in the same setting. 
The number of patients with CSLs are summarized in the Table 2. Of the 332 patients who underwent colonoscopy, 42 (12.7\%) had CSLs, with the majority of these being either malignancy (16) or high-grade adenomas (21). There were also 3 new cases of IBD found. Of the 258 patients who underwent EGD, 49 $(19.0 \%)$ had CSLs (4 patients had more than one CSL). These lesions were mainly vascular ectasias, peptic ulcer disease, celiac disease, severe erosive esophagitis, and esophageal varices. Overall, 91 patients (27.2\%) had a CSL in either EGD or colonoscopy, accepting that 76 patients did not undergo EGD. A total of 5 patients $(1.5 \%)$ had CSLs in both the upper and lower GIT.

Of the 243 patients who did not have CSLs identified on colonoscopy +/-gastroscopy, 75 (30.9\%) patients underwent further small bowel imaging to investigate a cause for IDA. An additional 9 patients underwent small bowel imaging despite having had a CSL identified in either EGD or colonoscopy. Characteristics of patients who did or did not undergo small bowel imaging are presented in Supplemental Table 1. Notably, a higher proportion of individuals underwent small bowel imaging if they had at least one other presenting complaint on initial evaluation ( $42.9 \%$ vs. $26.0 \%)$.

Of the 84 patients who had small bowel imaging studies, 68 had CTE, 13 had small bowel follow through, one had MRE, one had CT enteroclysis, and one had a push enteroscopy. Only one patient $(1.2 \%)$ in this group had a relevant CSL, which was identified as small bowel Crohn's disease. This patient was otherwise asymptomatic at the time of presentation. One patient had an incidentally discovered lung malignancy. One patient who had refused EGD was found to have severe erosive esophagitis on small bowel follow through.

\section{Discussion}

In this retrospective cross-sectional study of patients aged 50 years or older who underwent GIT investigations for IDA in the absence of overt bleeding, we found that small bowel imaging following a negative colonoscopy and gastroscopy carried a very low yield for diagnosing CSLs $(1.2 \%$ for relevant small bowel lesions). Moreover, no patients were identified as having small bowel neoplastic lesions. Additionally, we found that more than a quarter of patients had a CSL on either EGD or colonoscopy, reaffirming the utility of these investigations as part of the routine work-up for IDA, even in the absence of overt bleeding. EGD demonstrated a slightly higher yield in our cohort as compared to colonoscopy $(19.0 \%$ vs. $12.7 \%)$.

Overall, our results suggest that individuals over the age of 50 with isolated IDA in the absence of rectal bleeding frequently harbor a CSL in the upper or lower GIT, but rarely harbor significant small bowel lesions that are outside the reach of standard EGD and colonoscopy. While only $30 \%$ of patients with negative initial endoscopic investigations in our cohort ultimately underwent small bowel imaging, we did not identify any major differentiating characteristics between those who did or did not undergo small bowel imaging, suggesting that this subgroup may be reasonably representative of the total cohort. Importantly, the vast majority of the patients in our study underwent either CTE or small bowel followthrough, even though VCE is recommended as the procedure of choice in this setting. This may have 
lowered the yield of CSLs, particularly for vascular ectasias and small bowel Crohn's disease. However, we would not expect a major impact of small bowel imaging choice on the identification of small bowel neoplastic lesions, which would be the most concerning finding mandating therapeutic intervention in this setting. Nevertheless, our findings require confirmation in larger prospective studies.

Our findings differ considerably from other studies in the literature. A systematic review conducted by Koulaouzidis et al in 2012 that evaluated 1960 patients undergoing GIT investigations for IDA across 24 studies showed a diagnostic yield of $47 \%$ using VCE (ranging from $32 \%$ to $78 \%$ across studies) [9]. However, this review did not distinguish individuals with overt versus occult bleeding. The diagnostic yield of small bowel imaging specifically in those without overt bleeding is likely much lower, as evidenced by a study conducted by Cengic et al, in which MRE detected positive findings in $7.5 \%$ of patients with refractory iron deficiency anemia, including 2 patients with malignancy (1 adenocarcinoma, 1 lymphoma), 1 with Crohn's disease, and 1 with antral gastritis that was later seen on repeat gastroscopy [10]. To our knowledge, no other studies have reported detection rates of CSLs in small bowel investigations among patients with IDA without overt bleeding. Collectively, the findings from our study and those in the literature suggest that pursuing further small bowel investigations is mainly useful in patients with IDA and overt GIT bleeding.

Many societal guidelines continue to recommend small bowel imaging studies for investigation of IDA following negative EGD and colonoscopy $[13,14,15]$, albeit some do not make a clear distinction between those with or without overt GIT bleeding. Our findings do not support a role for routine small bowel radiographic imaging, specifically CTE or small bowel follow through, in persons with new-onset undiagnosed IDA without overt GIT bleeding. Notably, there may still be a role for VCE and/or DBE in this setting, and possibly even MRE, although this requires further study. Conversely, these resources are costly and may not be available in all centers.

There are a number of limitations to our study. We used a convenience sample of patients who underwent endoscopy for investigation of IDA, which may or may not be representative of all persons who develop IDA in society. Furthermore, as procedural indication was ascertained retrospectively and based primarily on endoscopists' impression, patients may have been misclassified as either having or not having IDA. Moreover, we did not have laboratory confirmation of IDA in many cases and included some cases of microcytic anemia, which may not have reflected IDA in all situations. We included patients with microcytic anemia (without iron studies) and iron deficiency without documented anemia to capture the breadth of patients in the spectrum of IDA. Notably, many patients were already taking iron supplementation by the time of their procedure, which would have made laboratory investigations difficult to interpret in these patients.

Additionally, as the procedural algorithm for investigation of IDA was not protocolized at our centre during the study period, we relied on whatever investigations were performed to draw inferences on the utility of these tests, which may not have been accurate in all cases, particularly for small bowel imaging (as only $30 \%$ of patients received this test). Reporting of these studies was also not standardized and 
may have resulted in variable detection and interpretation of findings. Moreover, gold standard small bowel imaging (VCE or DBE) was not used in the vast majority of patients in our study, and so we are unable to draw any inferences regarding the utility of these tests for investigation of IDA. Furthermore, as all patients were ascertained from a single referral centre, our findings may not be generalizable to the broader population of persons that undergo investigation for IDA. Finally, our sample size was relatively small and, therefore, our estimates may be imprecise. Ultimately, large prospective studies are required to confirm or refute our findings and to provide more precise estimates of rates of CSLs in various segments of the GIT in this setting.

\section{Conclusion}

In summary, we found that upper and lower GIT endoscopy are valuable tools for investigating persons aged 50 years or older with new-onset undiagnosed IDA without overt GIT bleeding, whereas CTE and small bowel follow through have limited utility in this setting. As this was a retrospective study from a single tertiary care centre, our findings require confirmation in larger prospective studies of wellcharacterized cohorts. Furthermore, the utility of VCE and/or DBE in this setting as well as factors associated with higher risk of harbouring CSLs of the GIT require further investigation. In a world with constantly growing demands on health care resources, optimizing the application of diagnostic tests in this setting is an important step towards efficient resource allocation.

\section{References}

1. Zuckerman GR, Prakash C, Askin MP, Lewis BS. AGA technical review on the evaluation and management of occult and obscure gastrointestinal bleeding. Gastroenterology 2000; 118: 201-21.

2. Kepczyk T, Kadakia SC. Prospective evaluation of gastrointestinal tract in patients with irondeficiency anemia. Dig Dis Sci 1995; 40: 1283-9

3. Evans JA, Chandrasekhara V, Chathadi KV, Decker GA, Early DS, Fisher DA et al. The role of endoscopy in the management of premalignant and malignant conditions of the stomach. Gastrointestinal Endoscopy 2015; 82(1): 1-8.

4. ASGE guideline. The role of endoscopy in the patient with lower GI bleeding. Gastrointestinal Endoscopy. 2014; 79(6): 875-85.

5. Goddard AF, James MW, McIntyre AS, Scott BB. Guidelines for the management of iron deficiency anaemia. British Society of Gastroenterology. Gut 2011; 60: 1309-16.

6. Bull-Henry K, Al-Kawas FH. Evaluation of occult gastrointestinal bleeding. American Family Physician. 2013; 87(6): 430-6.

7. Sheba E, Farag A, Aref W, Elkholy S, Ashoush O. Double-balloon enteroscopy (DBE) in patients presenting with obscure gastrointestinal bleeding (OGIB). Arab Journal of Gastroenterology. 2017; 18: 228-33. 
8. Frye JM, Hansel SL, Dolan SG, Fidler JL, Wong Kee Song LM, Barlow JM, Smyrk et al. NSAID enteropathy: appearance at CT and MR enterography in the age of multi-modality imaging and treatment. Abdominal Imaging. 2015; 40: 1011-25.

9. Koulaouzidis A, Rondonotti E, Giannakou A, Plevris JN. Diagnostic yield of small-bowel capsule endoscopy in patients with iron-deficiency anemia: a systematic review. Gastrointestinal Endoscopy. 2012; 76(5): 983-92.

10. Cengic I, Tureli D, Aydin H, Bugdayci O, Imeryuz N, Tuney D. Magnetic resonance enterography in refractory iron deficiency anemia: a pictoral overview. World Journal of Gastroenterology. 2014; 20(38): 14004-9.

11. Němec L, Fabian P, Tomášek J, Jarkovský J, Šefr R, Fiala L. Malignant tumors of the small bowel. Sozhl Chir. 2017; 96(6): 252-9.

12. Chung C-S, Tai C-M, Huang T-Y, Chang C-W, Chen K-C, Tseng C-M. Small bowel tumors: a digestive endoscopy society of Taiwan (DEST) multicenter enteroscopy-based epidemiologic study. Journal of the Formosan Medical Association. 2017

13. Gerson LB, Fidler JL, Cave DR, Leighton JA. ACG clinical guideline: diagnosis and management of small bowel bleeding. Am J Gastroenterol. 2015; 110: 1265-87.

14. Enns RA, Hookey L, Armstrong D, Bernstein CN, Heitman SJ, Teshima CT et al. Clinical practice guidelines for the use of video capsule endoscopy. Gastroenterology. 2017; 152: 497-14.

15. Pennazio M, Spada C, Eliakim R, Keuchel M, May A, Mulder CJ. Small-bowel capsule endoscopy and device-assisted enteroscopy for diagnosis and treatment of small-bowel disorders: European Society of Gastrointestinal Endoscopy (ESGE) Clinical Guideline. Endoscopy. 2015; 47: 352-76.

16. Jeon SR, Jin-Oh K, Gun KH, Hee LT, Jun-Hyung C, Ju PE. Is there a difference between capsule endoscopy and computed tomography as a first-line study in obscure gastrointestinal bleeding? Turk J Gastroenterol. 2014; 25: 257-63.

17. He B, Gong S, Hu C, Fan J, Qian J, Huang S, Cui L, Ji Y. Obscure gastrointestinal bleeding: diagnostic performance of 64-section multiphase CT enterography and CT angiography compared with capsule endoscopy. Br J Radio. 2014; 87(1043): 20140229. doi: 10.1259/bjr.20140229

18. Gonzalez-Suarez B, Rodriguez S, Ricart E, Ordas I, Rimola J, Diaz-Gonzalez, et al. Comparison of capsule endoscopy and magnetic resonance enterography for the assessment of small bowel lesions in Crohn's Disease. Inflamm Bowel Dis. 2018; 24(4): 775-80.

19. Iron deficiency anaemia: assessment, prevention, and control. WHO. 2001. Accessed online at: http://www.who.int/nutrition/publications/en/ida_assessment_prevention_control.pdf

20. D'Hoore W, Sicotte C, Tilquin C. Risk adjustment in outcome assessment: the Charlson comorbidity index. Methods Inf Med. 1993; 32(5): 382-7.

\section{Tables}




\begin{tabular}{|c|c|}
\hline \multicolumn{2}{|c|}{ Table 1 - Baseline Characteristics of Study Patients } \\
\hline Sex & \\
\hline Male & 167 \\
\hline Female & 167 \\
\hline \multicolumn{2}{|l|}{ Age } \\
\hline $50-59.9$ & 99 \\
\hline $60-69.9$ & 100 \\
\hline $70-79.9$ & 87 \\
\hline $80+$ & 48 \\
\hline \multicolumn{2}{|l|}{ Charlson Co-Morbidity Index } \\
\hline 0 & 110 \\
\hline 1 & 90 \\
\hline 2 & 56 \\
\hline 3 & 31 \\
\hline $4+$ & 47 \\
\hline \multicolumn{2}{|c|}{ Number with Additional Symptoms } \\
\hline Weight Loss & 32 \\
\hline Altered Bowel Habits & 32 \\
\hline Abdominal Pain & 14 \\
\hline GERD & 17 \\
\hline Dysphagia & 12 \\
\hline Bloating & 9 \\
\hline Nausea & 3 \\
\hline Vomiting & 4 \\
\hline Dyspepsia & 10 \\
\hline
\end{tabular}




\begin{tabular}{|c|c|}
\hline \multicolumn{2}{|c|}{ Table 2 - Procedures and Clinically Significant Findings } \\
\hline Procedure & Number of Clinically Significant Lesions \\
\hline EGD $(N=258)$ & 53 \\
\hline Malignancy & 1 \\
\hline Vascular Ectasia & 11 \\
\hline Peptic Ulcer Disease & 15 \\
\hline Severe Erosive Esophagitis & 8 \\
\hline Gastric/Esophageal Varices & 9 \\
\hline Celiac Disease & 8 \\
\hline GIST & 1 \\
\hline Colonoscopy $(\mathrm{N}=332)$ & 42 \\
\hline Malignancy & 16 \\
\hline High Grade Adenoma & 21 \\
\hline IBD & 3 \\
\hline Vascular Ectasia & 1 \\
\hline Large Rectal Varices & 1 \\
\hline Small Bowel Imaging $(\mathrm{N}=84)$ & 2 \\
\hline IBD & 1 \\
\hline Lung Malignancy & 1 \\
\hline
\end{tabular}

\section{Supplementary Table 1}


Supplementary Table 1 - Baseline Characteristics of Study Patients who Received Small Bowel Imaging vs. Patients who did not Receive Small Bowel Imaging

\begin{tabular}{|c|c|c|}
\hline & $\begin{array}{l}\text { Received Small Bowel } \\
\text { Imaging, } \mathrm{N}=84 \\
\text { (\%) }\end{array}$ & $\begin{array}{c}\text { Did not Receive Small Bowel } \\
\text { Imaging, } N=250 \text { (\%) }\end{array}$ \\
\hline \multicolumn{3}{|l|}{ Sex } \\
\hline Male & 38 (45.2\%) & $129(51.6 \%)$ \\
\hline Female & 46 (54.8\%) & 121 (48.4\%) \\
\hline \multicolumn{3}{|l|}{ Age } \\
\hline $50-59.9$ & 29 (34.5\%) & 70 (28.0\%) \\
\hline $60-69.9$ & 19 (22.6\%) & 81 (32.4\%) \\
\hline $70-79.9$ & $21(25.0 \%)$ & $66(26.4 \%)$ \\
\hline $80+$ & 15 (17.9\%) & 33 (13.2\%) \\
\hline \multicolumn{3}{|l|}{$\begin{array}{l}\text { Charlson Co-Morbidity } \\
\text { Index }\end{array}$} \\
\hline 0 & 30 (35.7\%) & $80(32.0 \%)$ \\
\hline 1 & $21(25.0 \%)$ & $69(27.6 \%)$ \\
\hline 2 & $12(14.3 \%)$ & $44(17.6 \%)$ \\
\hline 3 & $8(9.5 \%)$ & $23(9.2 \%)$ \\
\hline $4+$ & 13 (15.5\%) & 34 (13.6\%) \\
\hline $\begin{array}{l}\text { Number with Additional } \\
\text { Symptoms }\end{array}$ & 36 (42.9\%) & 65 (26.0\%) \\
\hline Weight Loss & $9(10.7 \%)$ & $23(9.2 \%)$ \\
\hline $\begin{array}{c}\text { Altered Bowel } \\
\text { Habits }\end{array}$ & $11(13.1 \%)$ & 21 (8.4\%) \\
\hline Abdominal Pain & $4(4.8 \%)$ & $10(4.0 \%)$ \\
\hline GERD & $4(4.8 \%)$ & $13(5.2 \%)$ \\
\hline Dysphagia & $5(6.0 \%)$ & $7(2.8 \%)$ \\
\hline Bloating & $5(6.0 \%)$ & $4(1.6 \%)$ \\
\hline
\end{tabular}




\begin{tabular}{|l|c|c|} 
Nausea & $0(0 \%)$ & $3(1.2 \%)$ \\
\hline Vomiting & $2(2.4 \%)$ & $2(0.8 \%)$ \\
\hline Dyspepsia & $2(2.4 \%)$ & $8(3.2 \%)$ \\
\hline
\end{tabular}

